that evaluated C-BOP/BEP treatment of poorprognosis germ-cell tumors.

The C-BOP/BEP schedule could be more appropriate than BEP for very sick patientsthose who have a large tumor burden at diagnosis and are, therefore, at high risk of neutropenia and sepsis-because the proportion of myelotoxic drugs used in the first few weeks of a C-BOP/BEP regimen is lower than that used in BEP.

Phase III trials are planned that will compare BEP and C-BOP/BEP directly.

Caroline Barranco

Original article Fosså SD et al. (2005) Intensive induction chemotherapy with C-BOP/BEP for intermediate- and poorrisk metastatic germ-cell tumours (EORTC trial 30948). Br J Cancer 93: 1209-1214

\section{Post-TURP stricture could be prevented by COX2 inhibitor therapy}

Urethral stricture is one of the main complications of transurethral resection of the prostate (TURP), occurring in 4-29\% of cases. Several methods have been tried to reduce the incidence of post-TURP stricture, but a need for a noninvasive, effective treatment remains. A new study indicates that post-TURP administration of a cyclo-oxygenase 2 (COX2) inhibitor could not only provide short-term benefits, in terms of peak urinary flow rate, but might also prevent the occurrence of postoperative stricture.

Sciarra et al. prospectively recruited 96 consecutive men who underwent TURP for benign prostatic hyperplasia at a single center. The men were randomly allocated to receive either rofecoxib $25 \mathrm{mg}$ daily (for 20 days, starting when the catheter was removed) or no COX2 inhibitor. Men who had received rofecoxib showed a statistically significant $(P<0.0001)$ improvement in their peak urinary flow rate by 1 month follow-up, compared with men who received no COX2 inhibitor. Importantly, urethral stricture occurred only in men who did not receive rofecoxib (17\%). No serious adverse events were associated with rofecoxib therapy during 1 year of follow-up.

Despite the limitations of the study - it was unblinded and lacked a placebo arm, and the study drug has since been withdrawn by the FDA - the authors suggest that other COX2 inhibitors might prove equally effective, and call for larger, controlled trials.

Caroline Barranco

Original article Sciarra A et al. (2005) Use of cyclooxygenase-2 inhibitor for prevention of urethral strictures secondary to transurethral resection of the prostate. Urology 66: 1218-1222

\section{Tumorigenic prostate cancer stem cells}

The identification of prostate cancer stem cells constitutes a major breakthrough in the understanding of tumorigenesis and could lead to new therapies that attack the disease at its root.

Prostate tumors are composed of a mix of cell types, a tiny subset of which are thought to maintain the tumor's proliferative and malignant capacity. Collins et al. in the UK had previously isolated stem cells from healthy human prostates bearing the cell surface markers CD44 ${ }^{+}$/ $\alpha_{2} \beta_{1}$ hi CD133 ${ }^{+}$. Now, these investigators have used the same markers to isolate and characterize stem cells from primary and metastatic prostate tumors.

First, the team determined that a small population of stem cells could be isolated from 40 human prostate tumors, regardless of tumor grade. Cell cultures were established from six of the tumors, and the investigators showed that these stem cells were capable of proliferation, self-renewal, differentiation, and invasion in vitro.

On the basis of their findings, the authors predict that differentiation from an androgen-receptornegative stem cell population determines tumor heterogeneity and the androgensensitive phenotype of prostate cancer.

In addition to identifying new therapeutic targets, this work also sheds light on the ongoing controversy over the cellular origins of prostate cancer: only basal cells were able to self-renew and differentiate, indicating that it is these cells from which prostate cancer originates.

Experiments in vivo are now needed to further investigate the tumorigenic properties of prostate cancer stem cells.

Tamsin Osborne

Original article Collins AT et al. (2005) Prospective identification of tumorigenic prostate cancer stem cells. Cancer Res 65: 10946-10951 